\title{
Influence of Eco-driving on Fuel Consumption of Vehicles in the Following Traffic
}

\author{
Md. Abdus Samad Kamal ${ }^{1)}$ Masakazu Mukai ${ }^{2)}$ Junichi Murata ${ }^{3)}$ Taketoshi Kawabe $^{4)}$ \\ 1) The University of Tokyo, Institute of Industrial Science, \\ 4-6-1-Ce605 Komaba, Meguro-ku,Tokyo 153-8505, Japan (E-mail: maskamal@ieee.org) \\ 2)-4) Kyushu University, Graduate School of Information Science and Electrical Engineering. \\ 744 Motoka, Nishi-ku, Fukuoka 819-0395, Japan
}

Received on Jun 17, 2011

Presented at the JSAE Annual Congress on May 20, 2011

\begin{abstract}
Ecological (eco) driving features distinct driving behavior that aims at improving fuel economy of a car and consequently reducing carbon dioxide emissions. Various assistance systems have recently been developed to promote ecodriving among drivers. Through anticipation of the preceding traffic, an eco-driver smoothly regulates the velocity and headway of the car at some safe levels. Limited use of accelerator and brake often causes an eco-driven car slower than other vehicles and affects behavior of its following traffic greatly. This paper investigates the influence of eco-driving on the fuel consumption of vehicles in the following traffic. Performance of the eco-driven car and vehicles behind it are observed in various traffic flow conditions through simulation in AIMSUN microscopic traffic simulator.
\end{abstract}

KEY WORDS: Social system, energy-saving driving, traffic stream, Model predictive control [F1]

\section{Introduction}

Development of environmentally friendly vehicles is an important issue for realization of sustainable transportation systems. Besides various physical factors, fuel consumption and carbon dioxide emission are greatly influenced by driving styles $^{(1,2)}$. Depending on the situations on the roads, fuelefficient driving strategies are to anticipate what is happening ahead and drive with smooth acceleration and braking, regulate a safe headway, cruise at a steady velocity and decelerate slowly at the stops $^{(3)}$. For the optimal fuel economy, a driver has to anticipate the road traffic situations properly and pose perfect knowledge of the engine dynamics of his car, which is hardly attainable by a human driver. Therefore, the driver can be technologically assisted to drive in such a fuel efficient style. It is expected that through the advancement of sensing, communication and information technologies, such eco-driver assistance systems will widely be used in transportation systems in the future.

Driver assistance for eco-driving in various conceptual frameworks has emerged recently. Speculative features of ecodriving are available in the form of driving tips ${ }^{(3)}$. Some recently manufactured cars have an indicator that shows green 'ECO' mark to the driver when it consumes little or no fuel. A driver may find his driving as ecological only when he maintains a steady velocity at a reasonable level or brakes the car. Some car service providers have launched off-board eco-driving supports for their members in which, after driving records are sent to a telemetric data center for off-line analysis, the data center send advices to the driver for improving his driving style in the next time. Based on the past performance, they have proposed an onboard assistance system to motivate the driver for eco-driving by showing his comparative driving efficiency, his position in the fuel composition ranking(4). An optimal driving, proposed for maximizing fuel economy of a vehicle, determines the optimal way to accelerate from the rest to cruising speed, drives a block between stop signs, and cruises on hilly terrain while maintaining a given average speed ${ }^{(5)}$. A similar approach over an entire drive cycle is used to derive the optimal switching strategy when the vehicle speed is fixed to the drive cycle ${ }^{(6)}$. These dynamic programming approaches are not appropriate for online implementation as they require knowledge of the full drive cycle before the trip. The above optimal driving approaches mainly focus automatic driving of a vehicle without addressing the influence of other vehicles in the traffic.

A new assisting approach, called the Ecological Driver Assistance System (EDAS), has been proposed in ${ }^{(7)}$. Using current road traffic information the EDAS anticipates the future states of vehicles using their simplified dynamic models, and based on the anticipated future states and the fuel consumption model of the engine, it calculates the optimal vehicle control input to assist the driver. An enhanced version of eco-driving system has been presented recently ${ }^{(8)}$. The system measures 


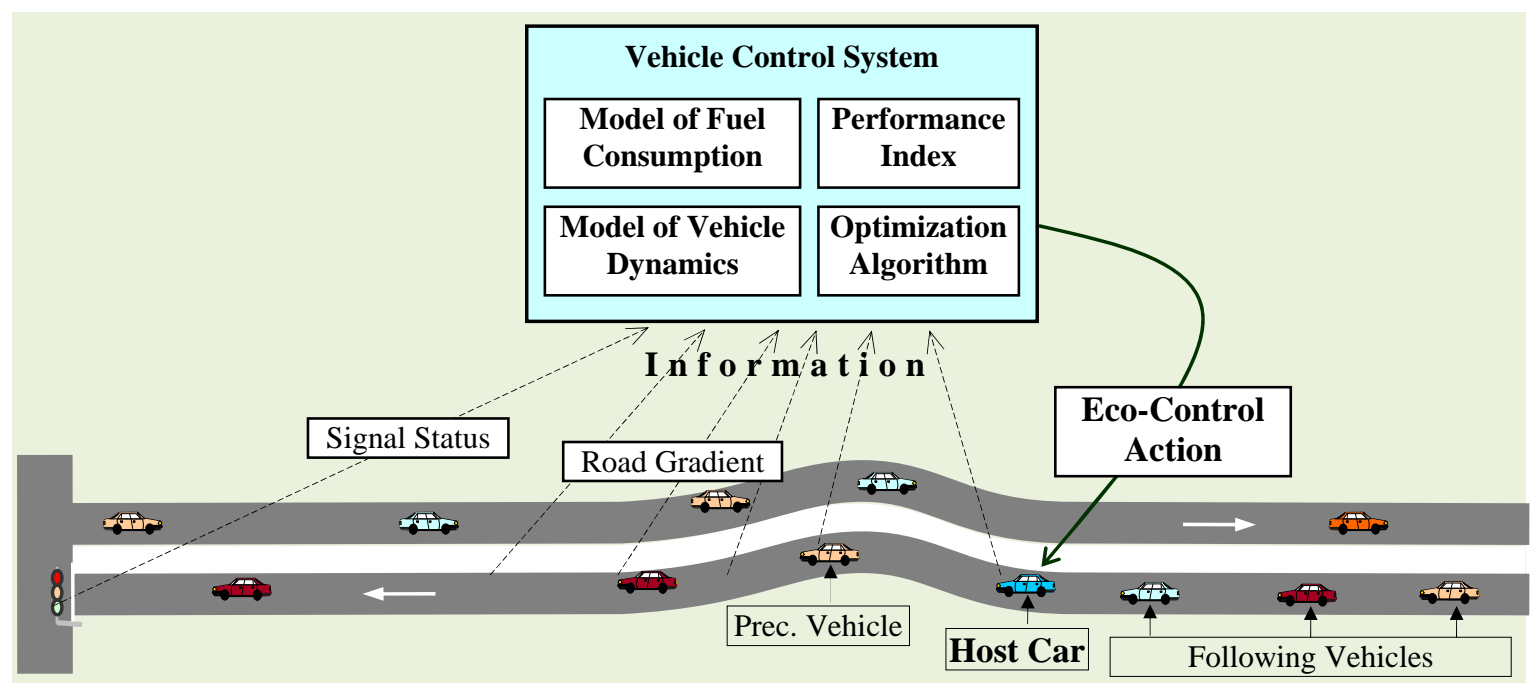

Fig. 1 The fundamental concept of an eco-driving system to control the host car.

information of a vehicle, preceding vehicle, road gradient and status of the traffic signal ahead. After receiving relevant information, it calculates optimal vehicle control inputs using model predictive control. Performance of the eco-driving system including its computational soundness and time, fuel saving aspects, has been evaluated on typical crowded urban roads.

Research on eco-driving mainly focuses either on its technological development or its benefits. However, the effects of eco-driving on other vehicles have not been investigated explicitly. In this paper, eco-driving system proposed in ${ }^{(8)}$ is taken to evaluate its influences on the following traffic, in which the other vehicles are driven conventionally. On a pseudorealistic traffic environment in AIMSUN simulator, performance and characteristics of eco-driving and conventional driving are compared at various traffic flow conditions. For a long drive on urban roads, the influence of eco-driving on vehicles in the following traffic in terms of fuel economy or mileage and traveling time are investigated. It is found that in most cases the conventionally following vehicles also improve their fuel consumption noticeably while running closely behind an ecodriving car.

The rest of the paper is organized as follows. Section 2 reviews an eco-vehicle driving system including vehicle dynamics and implementation of model predictive control. Section 3 describes the simulation results. Finally, Section 4 concludes the results of the paper.

\section{Ecological Vehicle Driving System}

This section describes the fundamental concept of ecodriving and reviews the modeling and control frameworks for realizing an eco-driving system ${ }^{(8)}$. Generally, eco-driving means maneuvering a vehicle in distinct styles, which is intended to enable energy conservation, reduce carbon dioxide emissions and noises, and ensure safe and comfortable driving. Aggressive driving, with speeding, rapid acceleration and braking, wastes energy considerably. Avoiding excessive acceleration and braking and cruising at the optimal velocity require proper anticipation of the traffic ahead. Fig. 1 shows the concept of the vehicle control system for eco-driving using model predictive control $^{(8)}$. The system measures the road gradient, the states of vehicles and traffic signal ahead. Various sensory mechanisms, such as GPS, on-board cameras or laser sensors can be used for this purpose. The road gradient information can be obtained through digital road maps ${ }^{(9)}$. Using inter-vehicle communication, a vehicle can receive information instantly when the driver of the preceding vehicle presses or releases the accelerator or brake pedal. Through communication with road infrastructure, status of traffic signals and their exact timing can also be obtained. Using measured information, the future states and fuel consumption of the vehicles are anticipated. With a suitable performance index the optimal control input necessary for a safe and fuel efficient driving is generated in a model predictive control framework. Once the optimal input is computed, using some sort of human interface, the control input can be transmitted to the driver or directly to the engine using an adaptive cruise control (ACC) system.

\subsection{Vehicle Dynamics}

For controlling a car, called the host car, its dynamics and dynamics of its preceding vehicle are modeled. The effects of other vehicles ahead and traffic signal are considered indirectly 
through the prediction model of the preceding vehicle. Dynamics of a nonlinear vehicle control system are represented by

$$
\dot{x}(t)=f(x(t), u(t), q(t))
$$

where the vector $x=\left[x_{h}, v_{h}, x_{p}, v_{p}\right]^{T}$ includes the positions and velocities of the host and preceding vehicle, respectively. The suffix $h$ and $p$ represent the host and the preceding vehicle, respectively. The input vector $u$ includes host vehicle input $u_{h}$, and $q(t)$ is a time varying parameter. The velocity of the host vehicle is related by

$M \frac{d v_{h}(t)}{d t}=F_{h}^{T}(t)-F_{h}^{R}(t)$

where $M, F_{h}^{T}$ and $F_{h}^{R}$ are the equivalent mass of the vehicle and its rotating parts, the traction force, and the sum of all motion resistance forces, respectively. The resistance forces including aerodynamic drag, rolling and gradient forces which are expressed by

$$
F_{h}^{R}=\frac{1}{2} C_{D} \rho_{a} A_{V} v_{h}^{2}+\mu M g \cos \theta\left(x_{h}\right)+M g \sin \theta\left(x_{h}\right)
$$

where $C_{D}, \rho_{a}, A_{V}, \mu$, and $\theta\left(x_{h}\right)$ are the drag coefficient, air density, frontal area of the vehicle, rolling resistance coefficient and road slip angle as a function of position $x_{h}$, respectively. The traction force is related by the mass of the vehicle and its control input as $F_{h}^{T}=M u_{h}$. The road gradient angle $\theta\left(x_{h}\right)$ is usually very small, therefore for computational simplicity it is approximated as $\sin \theta\left(x_{h}\right) \approx \theta\left(x_{h}\right)$, and $\cos \theta\left(x_{h}\right) \approx 1.0$.

The movement of the host vehicle depends on the presence of an adjacent preceding vehicle. It is assumed that the preceding vehicle is not influenced by the host vehicle, and its position $x_{p}$, velocity $v_{p}$ and acceleration $q=a_{p}$ can be measured at real time $t$. At $t_{1}^{\prime}$, the acceleration of the preceding vehicle at $t_{2}^{\prime}=t_{1}^{\prime}+\Delta t$ in the prediction horizon is estimated as follows.

$$
\begin{aligned}
q\left(t_{2}^{\prime}\right) & =f_{P V}\left(q\left(t_{1}^{\prime}\right), v_{p}\left(t_{1}^{\prime}\right)\right), \\
& = \begin{cases}q\left(t_{1}^{\prime}\right), & \left\{0<v_{p}\left(t_{1}^{\prime}\right)<v_{m}\right. \\
0, & \{\text { otherwise }\} .\end{cases}
\end{aligned}
$$

The traffic control signal is also taken into account by introducing a dummy preceding vehicle. In the case of a red or yellow signal at the end of a section (intersection), a dummy vehicle is assumed to be idling at the stopping point. In this way, the signaling system is considered in the decision making without any change in the problem formulation. Considering the above details of the host and preceding vehicle, the state equation (1) of the vehicle control problem can be rewritten as follows

$$
f(x, u, q)=\left[\begin{array}{c}
v_{h} \\
-\frac{1}{2 M} C_{D} \rho_{a} A_{V} v_{h}^{2}-\mu g-g \theta\left(x_{h}\right)+u_{h} \\
v_{p} \\
a_{p}
\end{array}\right] .
$$

\subsection{Fuel Consumption Estimation}

Efficiency of a vehicle at any driving conditions depends on the torque and rotational speed of the engine ${ }^{(10)}$. Exact derivation of the fuel consumption equation could be very complex. Instead, an approximate and differentiable function in terms of velocity and acceleration is used to estimate fuel consumption of a vehicle for eco-driving. The torque-speed-efficiency characteristics of an engine of a typical vehicle equipped with continuously variable transmission was constructed in such a way that the estimated fuel consumption on $10 \cdot 15$ mode matches the catalogue mileage of $17.2[\mathrm{~km} / \mathrm{l}]^{(9)}$. Fuel consumption data for sufficient sampled values of the velocity and acceleration are obtained from the engine efficiency characteristics map, and the fuel consumption rate $f_{\text {total }}[\mathrm{ml} / \mathrm{s}]$ is approximated using a polynomial function as

$$
\begin{aligned}
f_{\text {total }} & =f_{\text {cruise }}+f_{\text {acceleration }} \\
& =b_{3} v^{3}+b_{2} v^{2}+b_{1} v+b_{0}+\hat{a}\left(c_{2} v^{2}+c_{1} v+c_{0}\right)
\end{aligned}
$$

where $\hat{a}=a_{V}+a_{\theta}$ is the sum of the apparent acceleration of the vehicle $a_{V}$ and acceleration required internally to counteract the decelerating force due to the road slope, $a_{\theta}=g \theta(x)$. The fuel consumption rate at a steady velocity is represented by $f_{\text {cruise }}$, and the additional consumption due to the presence of acceleration is given by $f_{\text {acceleration }}$. It is assumed that if the vehicle input $u_{h} \leq 0$, no fuel is consumed in the engine. Details of the above fuel consumption estimation method are described in Kamal et $\mathrm{al}^{(9)}$.

\subsection{Model Predictive Control}

In eco-driving using model predictive control, at any time, the system measures the states of vehicles and traffic signal ahead and calculates a set of optimal control inputs required for a safe and fuel efficient travel in the prediction horizon. A symmetric constraint for $u_{h}$ using a dummy input $u_{d}$ is considered as $\left(u_{h}^{2}+u_{d}^{2}-u_{m x}^{2}\right) / 2=0$. For this problem, the performance index is chosen to have the following form 


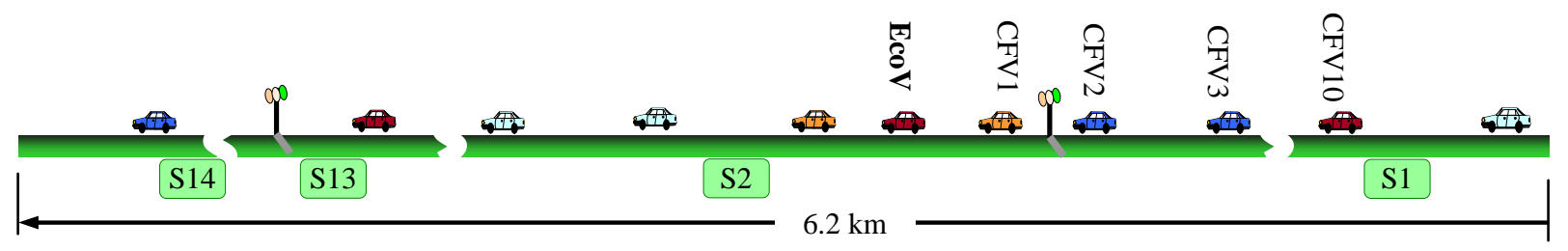

Fig. 2 An image of the road network in AIMSUN simulator used for observation of eco-driving and its influence on other traffic.

$\min _{u} J=\int_{t}^{t+T} L(x(\tau), u(\tau), q(\tau)) d \tau$

The cost function for eco-driving is defined as

$$
\begin{array}{r}
L(x, u, q)=w_{1}\left(\frac{f_{\text {cruise }}}{v_{h}}\right)+w_{2}\left(\frac{1}{2}\left(a_{V}+g \theta\left(x_{h}\right)\right)^{2}\right)+ \\
w_{3}\left(\frac{1}{2}\left(v_{h}-V_{r}\right)^{2}\right)+w_{4}\left(\left(h_{t}^{*} v_{h}-\left(x_{p}-x_{h}+R_{0}\right)\right)^{2}\right)
\end{array}
$$

where $V_{r}$ is the speed limit on the road, and $h_{t}{ }_{t}$ represents the desired headway for safely following a preceding vehicle. The cost function $L$ consists of four terms. The first term, multiplied by a weight of $w_{1}$, represents the cruising fuel economy $(\mathrm{ml} / \mathrm{m})$. The second term with a weight of $w_{2}$ represents the cost of acceleration and braking. The third term recommends running at the road specified speed and its influence depends on weight $w_{3}$. The last term is to keep a safe clearance from the preceding vehicle, which is multiplied by a time varying weight $w_{4}$. At each step in the prediction horizon, these weights represent their relative contextual merits or eligibility in the subjective situations. Weight $w_{4}$ is time varying and calculated as $w_{4}=r e^{-\alpha\left(h_{t}-h_{t}^{*}\right)}$, where the time headway is obtained as $h_{t}=\left(x_{p}-x_{h}-R_{0}\right) / v_{h}$. Weight $w_{4}$ ensures a large penalty at a closing preceding vehicle, and negligible penalty $\left(w_{4} \approx 0\right)$ when the preceding vehicle is at a safe distance. The other weights are fixed at some suitable values.

At each sampling step, the system measures the states of the vehicle. Using Continuation and generalized minimum residual (C/GMRES) ${ }^{(11)}$ method it generates the vehicle control inputs by solving the above optimal control problem. At each time only the immediate input calculated by this way is fed to control the host vehicle, and the whole process is repeated throughout the driving course, except at idling time. Since C/GMRES method has less computational burden than any iterative method ${ }^{(11)}$. The details of the computation algorithm using C/GMRES method for ecodriving are given in ${ }^{(8)}$.

\section{Simulation}

The eco-driving system has been simulated by using the parameters $u_{m x}=2.0\left[\mathrm{~m} / \mathrm{s}^{2}\right], h_{t}^{*}=1.80[\mathrm{~s}]$, and $V_{m}=15.0[\mathrm{~m} / \mathrm{s}]$. Fuel consumption parameters are approximated as $b_{0}=0.1569$, $b_{1}=0.0245, b_{2}=-7.415 \times 10^{-4}, b_{3}=5.975 \times 10^{-5}, c_{0}=0.07224$, $c_{2}=0.09681$, and $c_{3}=0.001075$. The weights in the performance index are set at $w_{1}=110.0, w_{2}=7.70, w_{3}=0.30$, and the parameters of $w_{4}$ is set at $r=0.016$ and $\alpha=2.954$. The prediction horizon of $T=50$ [s] is split into $N=100$ steps of size $=0.5[\mathrm{~s}]$.

\subsection{AIMSUN Traffic Simulator}

An image of the road-network, notations of vehicles and scenario for observation are illustrated in Fig. 2. A test network of about $6.2 \mathrm{~km}$ consists of 14 sections (S1-S14) of single lane was constructed in AIMSUN simulator. The consecutive sections are connected through traffic control signals, which are synchronously set at 90 seconds cycle including 52 seconds for green and 2 seconds for yellow signal, with respect to the route. The roads have a speed limit of $50[\mathrm{~km} / \mathrm{h}]\left(V_{r}=13.89[\mathrm{~m} / \mathrm{s}]\right)$. The car 'EcoV' is controlled by the eco-driving system. The conventionally-controlled following vehicles in the traffic are marked by 'CFV1'-'CFV10'. The influence on the fuel consumption of the first (CFV1) to the tenth (CFV10) vehicles behind the eco-vehicle are observed. An extension of AIMSUN simulator was created using application program interface (API) to collect traffic data and control a vehicle from the outside of AIMSUN for implementing the eco-driving. An arbitrary vehicle at the first section (S1) of the network is selected as the host vehicle, and then it is controlled through API until it exits the last section S14 after traveling about $6.2 \mathrm{~km}$. The car following model implemented in AIMSUN is based on Gipps model ${ }^{(12,13)}$. Gipps model is one of the popular car-following models that represent human driving. In AIMSUN, values of the Gipps model parameters among vehicles are randomly sampled from a truncated normal distribution ${ }^{(13)}$. Therefore, the vehicles in the simulator exhibit different driving behavior similar to human drivers of different characteristics and preferences in the traffic. 


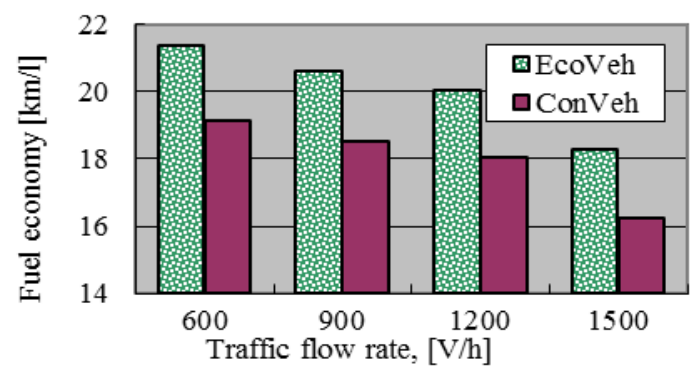

(a)

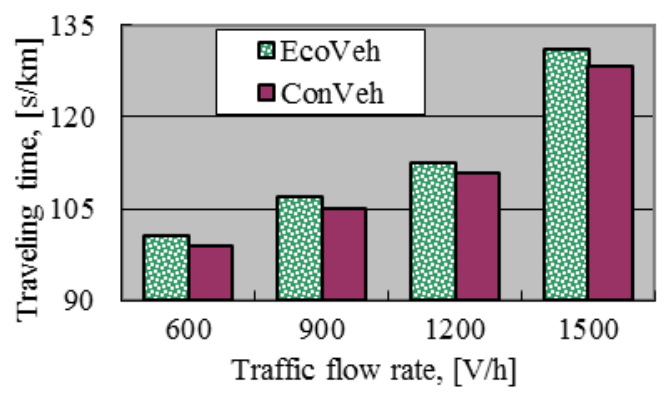

(b)

Fig. 3 Performance of eco-vehicles and conventional vehicle at various traffic flow conditions: (a) average fuel economy, and (b) average time for traveling one $\mathrm{km}$.

\subsection{Performance of Eco-driving Vehicle}

At first, performance of the eco-driving vehicle at various traffic flow conditions has been observed. Fuel consumption and traveling time of a vehicle have been monitored by controlling it using the eco-driving method. For the purpose of comparison, with the same initial conditions on the test route, performance of the vehicle have also been observed for conventional driving based on Gipps model. In these ways 35 driving cases have been observed independently using different vehicles in the traffic for a given traffic flow rate. Fig 3(a) shows the average fuel economy (mileage) of the eco-vehicles and conventional vehicles in four different traffic flow conditions. The improvement in fuel economy of the eco-vehicle is found in the range of $11 \sim 12 \%$ in these traffic flow conditions. The average traveling time per $\mathrm{km}$ at the corresponding traffic flow is shown in Fig. 3(b). Since the roads have single lane, conventional vehicles cannot overtake a slow vehicle on the road. It is found that the eco-vehicle requires $2 \%$ longer travel time than the conventional vehicle, and in return, it improves its fuel economy remarkably.

To comprehend and compare the behavior of eco-driving and conventional driving, the velocity and acceleration for a single signal cycle on the route are illustrated in Fig. 4. Fig. 4(a) shows the signal status: green, yellow and red, and Fig. 4(b) shows a typical conventional driving. In eco-driving, shown in Fig. 4(c), the car speeds up smoothly at the beginning, and it

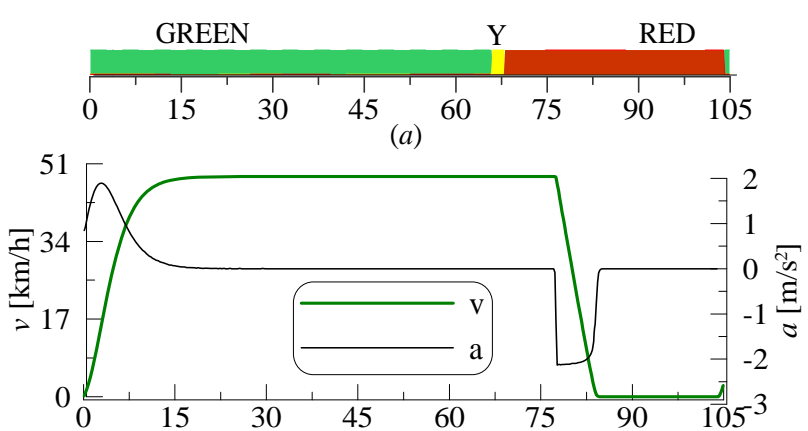

(b)

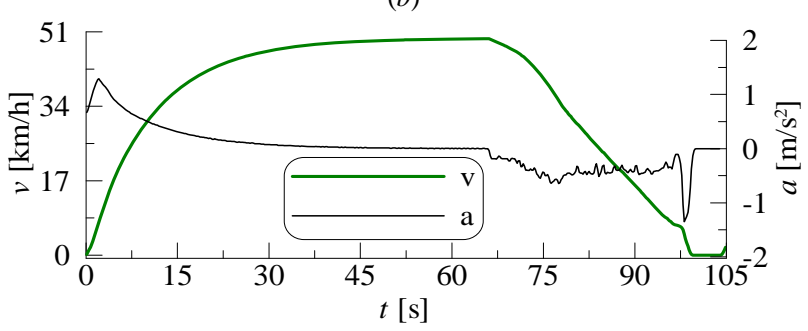

(c)

Fig. 4 (a) Traffic signal, (b) conventional driving, and (c) ecodriving.

starts decelerating as soon as the signal turns into yellow. In a similar fashion, the eco-driving car decelerates softly whenever there is a slowing preceding vehicle. In these ways it avoids waste of energy and improves the fuel economy.

\subsection{Effects on Vehicles in the Following Traffic}

The eco-driving car does not consider any following vehicles running behind it in deciding its control inputs. Although a following vehicle driven by the conventional method based on Gipps model, its speeding behavior is greatly influenced by the preceding eco-vehicle. Fig. 5(a) shows the average fuel economy of the vehicles in the following traffic. The results are observed at a traffic flow rate of 900 vehicles per hour. Fuel economy of the eco-driving vehicle ('EcoV') and its conventional following vehicles ('CFV1' to 'CFV10') are illustrated in the bar graph. The horizontal dotted line over the bars shows the average fuel economy when all the vehicles are driven conventionally. It can be observed that by the influence of eco-driving the first following vehicle (CFV1) has improved its mileage significantly, whereas the next three vehicles (CFV2CFV4) have also improved their mileage noticeably. Fig. 5(b) shows the average distance of the following vehicles from the host vehicle in two cases: in usual traffic (behind a conventional vehicle) and in behind an eco-driving host car. Influence on vehicles depends on how much and how long they closely run behind the eco-car on the road. Since the other following vehicles run far behind the host, their behavior is not much affected by the eco-driving car. 


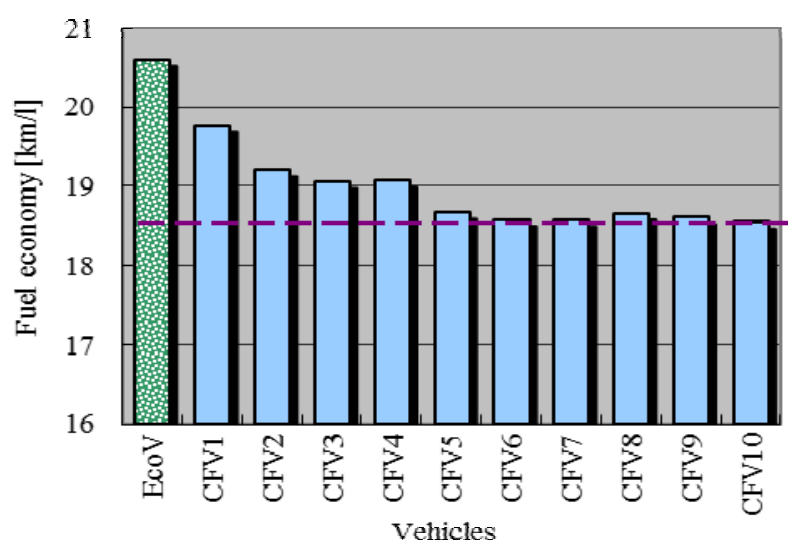

(a)

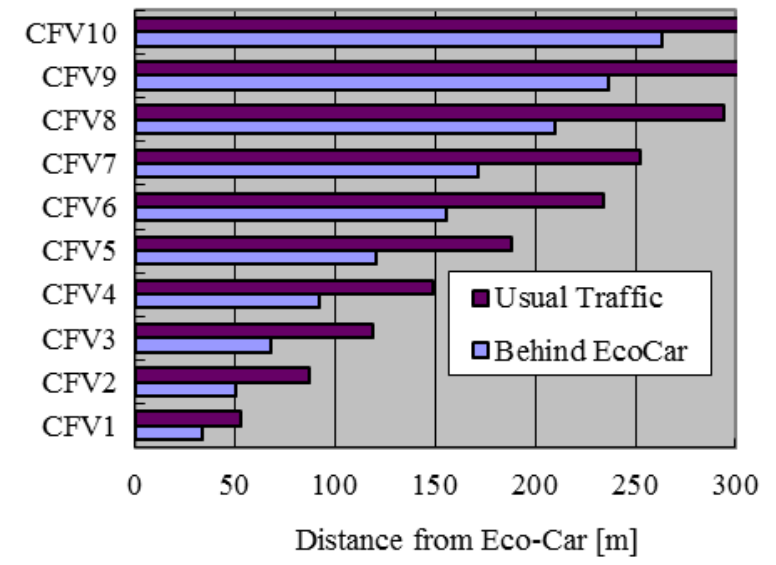

(b)

Fig. 5 Influence on vehicle $s$ in the following traffic a traffic flow rate 900 [V/h]: (a) average fuel economy, (b) average following distance.

The same observation has also been conducted at a traffic flow of 1500 vehicles per hour, which is shown in Fig. 6. Since at high traffic densities the vehicles follow with short headways from the preceding ones, the influence of individual eco-driving extended further in the behind. Up to the 9th following vehicles, noticeable improvement in their economy has been observed. The results show the average of 35 observations, in which about $80 \%$ cases, the fuel economy of the first following vehicle CFV1 is improved. Only in few cases the fuel economy worsens, and its reason is investigated. It is found that the conventionally driven vehicle, with a high desired speed and very aggreesive acceleration, often goes very close to the slow preceding vehicle (eco-car) and immediately has to brake. Therefore their unnecessary acceleration and braking causes waste of fuel. On the other hand, the vehicle with a low or moderate desired acceleration usually improves fuel economy while following an eco-driving car.

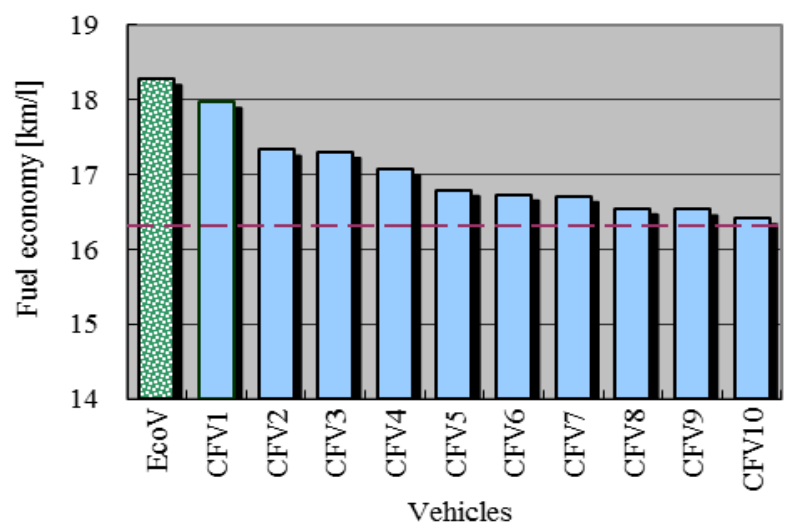

(a)

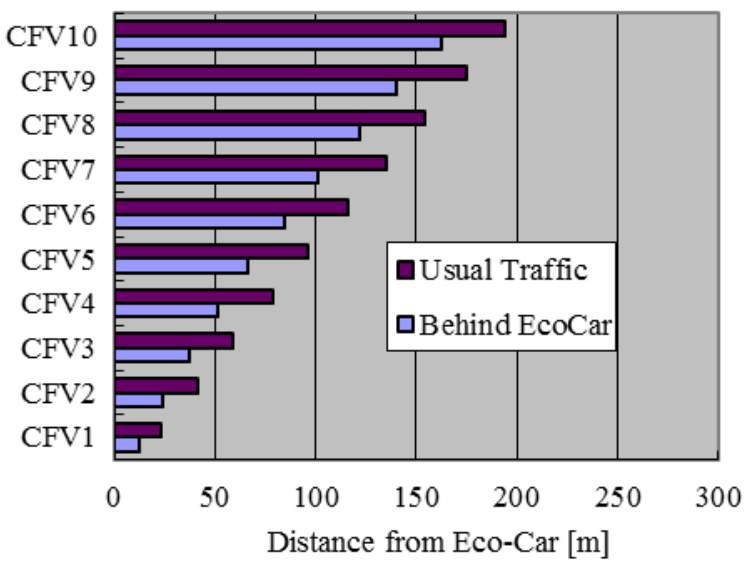

(b)

Fig. 6 Influence on vehicle $s$ in the following traffic at a traffic flow rate 1500 [V/h]: (a) average fuel economy, (b) average following distance.

\section{Conclusion}

This paper investigates the performance of eco-driving and its influence on the fuel consumption of the vehicles in the following traffic at various traffic flow conditions. Eco-driving using model predictive control and AIMSUN traffic simulator have been used in this evaluation. Although fuel economy and traveling time of the vehicles greatly affected by the traffic densities, the benefit of eco-driving over conventional driving remains significant at any traffic flow rates. By the influence of an eco-driving vehicle, fuel economy of vehicles in the following traffic has also been improved significantly. At a high traffic flow conditions the average separation between two consecutive vehicles is small, and more vehicles benefit by the eco-driving car. In the future, the influence of multiple eco-driving cars on multi-lane roads will be investigated. 


\section{References}

(1) J. Van Mierlo, G. Maggetto, E. Van de Burgwal and R. Gense: Driving style and traffic measures-influence on vehicle emissions and fuel consumption, Proc Inst Mech Eng D: J Automob Eng, vol. 218, p. 43-50 (2006).

(2) M.Taniguchi: Eco-driving and Fuel Economy of Passenger Cars, Proc. of Annual Meeting of IEE Japan, p.S21(5-8), (2008).

(3) FORD-WERKE: Ford eco-driving. Schneller schalten, weiter kommen. Cologne, (2003). http://www.ford-ecodriving.de/.

(4) K.Satou, R.Shitamatsu, M. Sugimoto, and E.Kamata: Development of an On-board Eco-driving Support System, Nissan Technical Review (in Japanese), no.65, p.68-71, (2009).

(5) J.N. Hooker: Optimal driving for single-vehicle fuel economy, Transportation Research Part A: vol. 22, no.3, p.183201, (1988).

(6) F. Kirschbaum, M.Back, M.Hart: Determination of the fueloptimal trajectory along a known route, in proc. of IFAC 15th Triennial World Congress, Barcelona, Spain, (2002).

(7) M.A.S. Kamal, M. Mukai, J. Murata and T. Kawabe: Ecological Driver Assistance System Using Model Based Anticipation of Vehicle-Road-Traffic Information, IET Journal of Intelligent Transportation Systems, vol. 4, no. 4, p.244 - 251 (2010).

(8) M.A.S. Kamal, M. Mukai, J. Murata and T. Kawabe: Model Predictive Control of Vehicles on Urban Roads for Improved Fuel Economy, to appear in IEEE Trans. on Intelligent Control Systems Technology, (2012).

(9) M.A.S. Kamal, M. Mukai, J. Murata and T. Kawabe: Ecological Vehicle Control on Roads with Up-Down Slopes,

IEEE Transactions on Intelligent Transportation Systems, vol. 12, no. 3, (2011).

(10) Society of Automotive Engineers Japan: Handbook of Automobile Technology. vol. 1, p.13-16. (in Japanese) (1990).

(11) T.Ohtsuka: A Continuation/GMRES Method for Fast Computation of Nonlinear Receding Horizon Control. Automatica, vol. 40, No. 4, p. 563-574, (2004).

(12) P.G. Gipps: A behavioural car following model for computer simulation, Trans. Res. Board, vol. 15-B, no. 5, p.403414 (1981)

(13) AIMSUN: AIMSUN NG User's Manual. Version 5.1.4, (2006), http://www.aimsun.com/. 